\title{
Early Cerebral Blood Flow Changes, Cerebrovascular Reactivity and Cortical Spreading Depolarizations in Experimental Mild Traumatic Brain Injury Model
}

\author{
Sahin HANALIOGLU1, Tural HIDAYATOV ${ }^{1}$, Ilkay ISIKAY ${ }^{1}$, Canan CAKIR AKTAS², Muge YEMISCI², Melike MUT¹ \\ ${ }^{1}$ Hacettepe University, Faculty of Medicine, Department of Neurosurgery, Ankara, Turkey \\ ${ }^{2}$ Hacettepe University, Institute of Neurological Sciences and Psychiatry, Ankara, Turkey
}

Corresponding author: Sahin HANALIOGLU hanalioglu@hacettepe.edu.tr, sahinhanalioglu@gmail.com

\section{ABSTRACT}

AIM: To investigate the spatiotemporal dynamics of early cerebral blood flow (CBF) changes, cerebrovascular reactivity (CVR), and vascular responses to cortical spreading depolarization (CSD) in an experimental mild traumatic brain injury (mTBI) model with laser speckle contrast imaging (LSCl) technique.

MATERIAL and METHODS: The weight-drop model was used to induce blunt head trauma. The mice were divided into two groups as mild TBI $(n=12)$, and sham $(n=6)$. The animals underwent continuous LSCl before and for 1 hour after trauma to evaluate the regional $\mathrm{CBF}$ changes, $\mathrm{CVR}$ in response to $\mathrm{CO} 2$, and CSD-associated vascular responses induced by pinprick.

RESULTS: Our minor blunt head trauma protocol induced CSD in only 2 (16.7\%) animals, which were excluded from further analyses. Of the remaining animals, $30 \%$ showed slight hyperemia following trauma, with mild ipsilateral hemisphere oligemia (15\%-20\% decrease in CBF) on average compared to baseline $(p=0.027)$ and contralateral hemisphere $(p=0.029)$. Maximal CBF decrease was measured in the peri-impact area $(24.1 \% \pm 5.1 \%)$. No significant difference was found between the sham and $\mathrm{mTBI}$ groups and two hemispheres of the mTBI group or pre/post-CSD periods of CO2 reactivity, as well as the characteristics of vascular CSD responses (net ischemia: $52.3 \% \pm 2.6 \%$ vs. $56.3 \% \pm 1.9 \%$ and prolonged oligemia duration $44.8 \pm 1.8 \mathrm{~min}$ vs. $49.8 \pm 2.3 \mathrm{~min}$ ).

CONCLUSION: The ipsilateral hemisphere, particularly in the peri-impact area, had mild hypoperfusion, within the first hour of minor blunt head trauma in mice. Nonetheless, mTBI does not alter CVR and vascular responses to an induced CSD, thus the overall CVR is largely preserved in mTBI without significant structural damage despite a mildly decreased CBF in the hyperacute phase.

KEYWORDS: Traumatic brain injury, Concussion, Cerebral blood flow, Cerebrovascular reactivity, Cortical spreading depolarization, Laser speckle contrast imaging

ABBREVIATIONS: AR: Autoregulation, CBF: Cerebral blood flow, CO2: Carbon dioxide, COSBID: Co-operative Studies on Brain Injury Depolarizations, CSD: Cortical spreading depolarization, CVR: Cerebrovascular reactivity, ICP: Intracranial pressure, H/E: Hematoxylin-eosin, K+: Potassium, LSCI: Laser speckle contrast imaging, mTBI: Mild traumatic brain injury, PaCO2: Partial pressure of carbon dioxide, rCBF: Regional cerebral blood flow, STX: Stereotaxic frame, TBI: Traumatic brain injury

Sahin HANALIOGLU (D) : 0000-0003-4988-4938 Tural HIDAYATOV (D) : 0000-0002-4143-3131

Ilkay ISIKAY (1):0000-0001-7790-4735
Canan CAKIR AKTAS 미 : 0000-0002-3401-3892

Muge YEMISCI $\quad$ (1) : 0000-0002-1528-1998

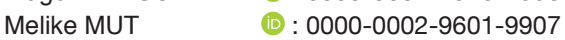




\section{- INTRODUCTION}

$\mathrm{T}$ Trauma is the most frequent cause of death under the age of 45 years, and traumatic brain injury (TBI) is the leading cause of trauma-related death and disability $(3,21)$. Its socioeconomic burden to society is also enormous (38). The attempts to mitigate secondary injury mechanisms in TBI are the mainstay of the management (3). Severe TBI is responsible for a greater portion of the TBI-related mortality and morbidity, whereas mild $\mathrm{TBI}(\mathrm{mTBI})$ or concussions comprise its vast majority (85\%-90\%), thus their long-lasting health effects may have a greater global socioeconomic burden (14). Therefore, it is equally important to better understand the underlying pathophysiological processes of mTBI to develop diagnostic tools and therapeutic measures to mitigate its early and late consequences on brain health and quality of life $(4,20,28)$.

Cerebral blood flow (CBF) changes immediately occur after trauma and are the hallmarks of secondary injury processes of severe TBI $(26,34)$. Ischemia is a well-known secondary injury mechanism that worsens clinical outcomes (12). Similarly, cortical spreading depolarizations (CSD), which are also linked to spreading ischemia, are associated with poor outcomes in patients with $\mathrm{TBI}(16,23)$. Thus, recognizing and preventing these secondary injury processes is important.

Many imaging and measurement tools have been used to demonstrate CBF alterations in severe TBI; however, data on the dynamics of CBF changes following an $\mathrm{mTBI}$ is limited (29). Early CBF changes may in part account for obscure but relevant pathophysiological changes that lead to early (e.g., amnesia, transient loss of consciousness) and late (e.g., headaches, emotional lability, anxiety, depression) clinical consequences (7). However, studying the CBF dynamics in the hyperacute phase of mTBI using imaging methods, particularly in clinical settings, is challenging. Additionally, current imaging and monitoring tools are limited in their capacity to detect CBF changes with high sensitivity, specificity, and spatiotemporal resolution.

Current imaging and monitoring tools are insufficient in terms of measuring CBF changes from different regions of interest at once with high sensitivity and specificity and at high spatiotemporal resolution. Laser speckle contrast imaging (LSCl) is a powerful, non-invasive, and full-field optical imaging tool to visualize real-time blood flow changes that appears as a good alternative to the current techniques to overcome these problems (1). Hence, this study aimed to investigate early CBF changes, cerebral vascular reactivity (CVR), and vascular responses to CSD after $\mathrm{mTBI}$ in mice using in vivo full-field LSCI.

\section{MATERIAL and METHODS}

\section{Animals}

This study was approved by the institutional Ethics Committee for Animal Research (IRB Approval No: 2015/100-07, Date: 25.12.2015), and was conducted following the national and international guidelines for the use of animals in research. Swiss Albino mice weighing between 25 and $35 \mathrm{~g}$ were used for the study. The animals were individually housed in standard polycarbonate caging, under 12-h light-12-h dark cycle, with ad libitum access to food and water.

\section{Study Groups}

A total of 27 mice were used throughout the study. In the preliminary experiments, three different levels of trauma severity ( $n=3$ /group) were studied in nine animals to establish an optimal injury paradigm to study the spatiotemporal dynamics of cortical CBF changes using LSCI (Figure 1). The subsequent experiments used the mTBI model as the standard $\mathrm{TBI}$ technique to study cortical regional CBF changes with LSCl in mice with intact skulls. Later, a total of 18 animals were divided into two groups as Group 1: Sham $(n=6)$ and Group 2: $\mathrm{mTBI}(\mathrm{n}=12)$. In the respective experiments, animals in the sham and $\mathrm{mTBI}$ groups underwent the same procedures except for trauma.

\section{Experimental Protocol}

The experimental protocol of the study is presented in Figure 1. Briefly, under isoflurane anesthesia, the animals underwent baseline $\mathrm{LSCl}$, followed by trauma induction and then posttraumatic LSCI for CBF monitoring. During the LSCI, immediate CBF changes after trauma were monitored, and CVR and CBF responses to cortical spreading depression induction were measured. Dynamic spatiotemporal monitoring of hyperacute CBF changes (as early as 2-3 mins after trauma) detected early CSD occurrences (captured at/after the third phase of CSD) that are induced with the described mTBI protocol. We identified two animals having CSDs that are immediately induced with our mild trauma protocol (i.e., not induced by pinprick) and excluded them in the following analyses.

\section{Anesthesia and Surgical Procedures}

General anesthesia was induced with $5 \%$ isoflurane and maintained with $2 \%$ isoflurane throughout the experiment. Hindpaw and tail pinch reflexes were monitored to confirm the depth of anesthesia. The mice were placed in a stereotaxic frame (Digital Lab Standard Stereotaxic Frame, Stoelting, USA) in the prone position. A midline skin incision from between the eyes to the neck was made to expose the entire cranium (both frontal and parietal bones and occipital bone). Baseline LSCI images were obtained and recorded over the intact skull (i.e., no bone drilling or craniotomy was performed) for up to $5 \mathrm{~min}$. Following the baseline LSCI, the mice were removed from the frame and placed in custom-made trauma apparatus. Trauma procedure and post-traumatic LSCI and other interventions were described in detail below. The animals were allowed for spontaneous breathing and supplied with $2 \mathrm{~L} / \mathrm{min}$ of oxygen to maintain normoxemia, and vital signs, including pulse rate and oxygen saturation (V3304 Digital Table-Top Pulse Oximeter, SurgiVet, USA), were monitored throughout the experiment. A rectal temperature probe with homeothermic blanket (Harvard Apparatus Limited, UK) was used to keep the body temperature stable at approximately $37.0^{\circ} \mathrm{C} \pm 0.2^{\circ} \mathrm{C}$. The depth of anesthesia was continuously assessed with hindpaw, tail pinch, and corneal reflexes at 15 min intervals. At the end of the experiment, the skin incision was sutured and the animal was returned to the cage. 

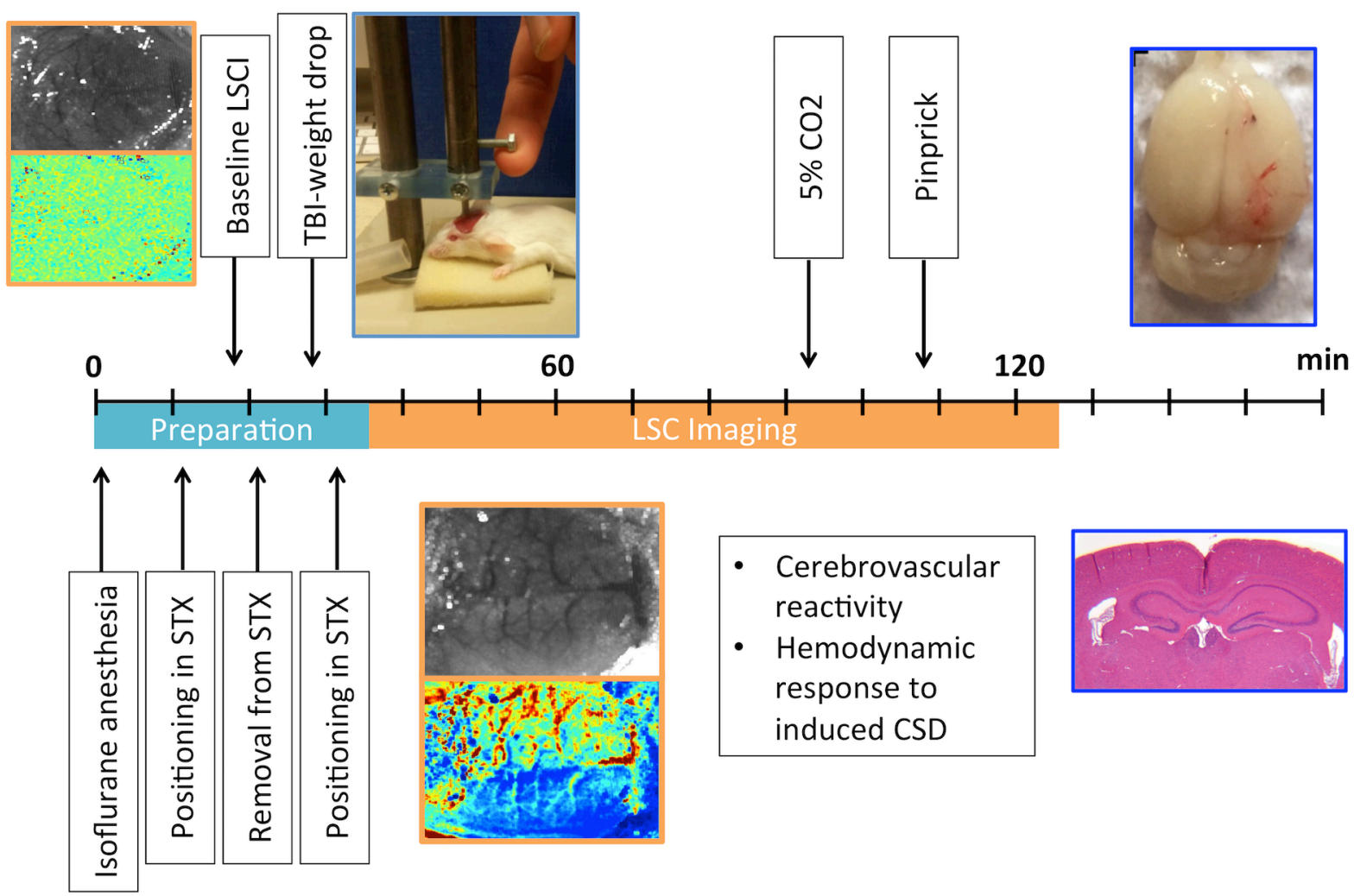

\section{- Cerebrovascular reactivity \\ - Hemodynamic response to induced CSD}

Figure 1: Experimental protocol of the study. CSD: Cortical spreading depolarization, $\mathbf{C O}_{2}$ : Carbon dioxide, LSCI: Laser speckle contrast imaging, STX: Stereotaxic frame, TBI: Traumatic brain injury.

\section{Traumatic Brain Injury}

Following the baseline CBF recording in the stereotaxic frame under isoflurane anesthesia, the mice were subjected to the sham procedure or mTBI using a weight-drop method to deliver TBI. The mice with TBI treatment were placed on a foam-covered platform without head fixation to result in a predominantly diffuse injury induced by a shearing force to mimic the real-world situation of minor blunt head trauma (i.e., concussion) (instead of a direct impact on the brain through craniotomy window). Additionally, the use of a stainless-steel disk helmet was not deemed feasible since dental cement or any type of adhesive to attach it to the skull would result in damage or opacification of the skull, thereby hindering the quality of $\mathrm{LSCl}$ recording.

A cylindrical steel weight ( $5 \mathrm{~mm}$ tip diameter and $67 \mathrm{~g}$ in weight) was freely dropped from a predetermined height onto the right parietal area. In the feasibility experiments ( $n=9$ animals), the same weight was dropped from different heights $(5 \mathrm{~cm}$, $7.5 \mathrm{~cm}, 10 \mathrm{~cm} ; \mathrm{n}=3 /$ group) to determine the optimal trauma paradigm for $\mathrm{LSCl}$, and $5 \mathrm{~cm}$ was chosen to produce $\mathrm{mTBI}$ without major structural abnormalities (no skull fracture or hemorrhages), which allowed post-traumatic $\mathrm{LSCl}$ recording in these animals. In the sham-treated control mice, the skull was exposed under anesthesia, and the animals were placed under TBI apparatus for a few seconds but the weight was not dropped. The animal was returned to a stereotaxic frame likewise.

\section{Laser Speckle Contrast Imaging}

After the trauma induction, the mice were immediately returned to the stereotaxic frame for subsequent LSCI to capture realtime CBF changes. The delay between trauma induction and the beginning of post-traumatic LSCI recording was $<3$ min (median: $2 \mathrm{~min}$ ) in all experiments.

The imaging setup is shown in Figure 2A-D. Before the imaging, the exposed skull was coated with a thin layer of immersion oil to prevent drying and improve the image quality. The skull was illuminated with a $785 \mathrm{~nm}$ laser diode (Thorlabs DL7140201, Thorlabs, United States) and the underlying vasculature and CBF changes were visualized with a stereomicroscope (Nikon SMZ 1000, Nikon, Japan) and "charge-coupled device" camera (Basler 602F, Basler Vision Technologies, Germany). LSCI was performed to continuously monitor CBF changes in the cerebral cortex under an intact skull as previously described (1). Continuous recording of relative CBF changes was performed for at least $60 \mathrm{~min}$. In the first $15 \mathrm{~min}$ after trauma, CBF changes were observed only without any intervention, which was then followed by the measurement of CVR- and CSD-related vascular changes sequentially.

\section{CVR Measurement}

Cortical CVR was assessed with $5 \% \mathrm{CO}_{2}$ inhalation. Thus, inhalation gases were replaced with $5 \% \mathrm{CO}_{2}$ and continued for 2 min while the cortical CBF changes were monitored via continuous LSCI. The cortical CVR was calculated as a 

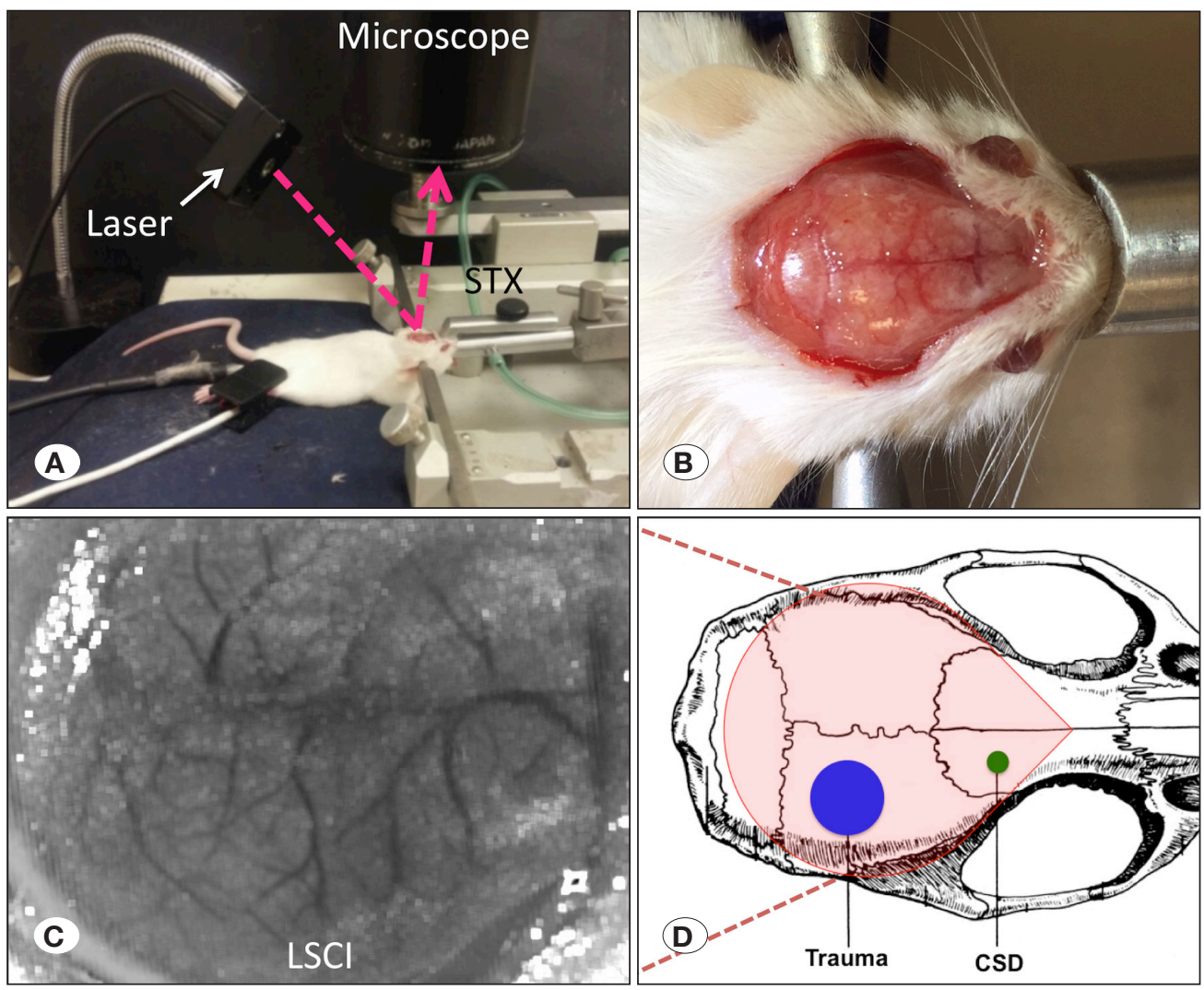

Figure 2: Setup for laser speckle contrast imaging. A) Laser is reflected on the exposed skull and captured via microscope and camera. B) Head is fixed in the stereotaxic frame (STX).

C) Laser speckle contrast imaging allows visualization of cortical vasculature and relative regional CBF changes. D) Trauma impact is applied on the right parietal cortex while a small frontal burr hole was used to induce CSD with a pinprick.

percent change in the baseline regional CBF. Each animal underwent at least two periods of $5 \% \mathrm{CO}_{2}$ testing (one before and one after the CSD induction).

\section{CSD Induction}

After recording the post-traumatic CBF changes and CVR to $5 \% \mathrm{CO}_{2}$ inhalation, a small burr hole was opened in the right frontal bone using a high-speed drill, and a CSD was induced with a pinprick through this burr hole. The cortical CBF response to pinprick was monitored to indirectly analyze the characteristics of the CSD via associated CBF changes.

\section{Cerebral Blood Flow Measurements}

Raw speckle contrast images of the cortex were acquired at 15-second intervals and recorded in the Speckle software. ImageJ 1.48 v software (NIH, USA) was used to analyze $\mathrm{LSCl}$ images to calculate relative CBF changes in the tissue. After importing all recordings of an animal, a square-shaped region of interest $(0.5 \times 0.5 \mathrm{~mm})$ in the cortex (avoiding major vessels and sutures) was selected to measure the CBF changes over time. All measurements were done relative to the baseline recordings. Pseudocoloring was performed to supplement the visualization of spatiotemporal dynamics of CBF changes in the cerebral cortex.

\section{Histopathologic Evaluation}

At 24 hours after trauma, the animals were anesthetized and perfused through the heart with $4 \%$ paraformaldehyde in a $0.1 \mathrm{~mol} / \mathrm{L}$ sodium phosphate buffer $(\mathrm{pH}: 7.4)$. In each animal, the brain was carefully removed from the skull and fixated in $4 \%$ paraformaldehyde solution, and stored at $4{ }^{\circ} \mathrm{C}$. Fixated brains were cut into five equal coronal slices of $2 \mathrm{~mm}$ from the olfactory bulbus to the cerebellum and all the pieces were embedded in paraffin. All the extracted fragments were fixed in $10 \%$ buffered neutral formalin for $48 \mathrm{~h}$. The alcohol concentrations were increased to ensure dehydration. For transparency, tissue samples were passed through xylol two times for $1 \mathrm{~h}$ and kept in pure paraffin for $2.5 \mathrm{~h}$ for paraffin acclimation.

The tissue samples were embedded in pure paraffin in Leica Eg1150H paraffin station and paraffin blocks were obtained. Sections of 5-micron thickness were taken from the obtained paraffin blocks. The prepared sections were left in an oven at $600{ }^{\circ} \mathrm{C}$ overnight and then deparaffinized by keeping them in xylol for $45 \mathrm{~min}$. The samples were rehydrated by passing them through graded alcohol solutions. After staining with hematoxylin-eosin $(\mathrm{H} / \mathrm{E})$, they were dehydrated by passing through graded alcohol series. Fibrin staining was also performed to detect microthrombi within the vessels.

\section{Statistical Analysis}

GraphPad Prism 9 software was used for statistical analysis. The descriptive analyses included mean \pm standard deviation for parametric and count (percentage) for categorical variables. The Kolmogorov-Smirnov test was used to test for normality. The Student's $t$ and Mann-Whitney $U$ tests were used to comparing two groups, and one-way analysis of variance and Kruskal-Wallis tests to compare multiple groups 
for parametric and nonparametric variables, respectively. The Wilcoxon signed-rank test was used to compare the measurements of the same group in two different periods. A $\mathrm{p}$-value of $<0.05$ was considered statistically significant.

\section{RESULTS}

mTBI Induces Hyperacute Transient Cortical Oligemia in The Ipsilateral Hemisphere

With the used experimental paradigm in this study, we can observe the hyperacute cortical CBF changes, even as early as at 3 min following mTBI. Most animals $(n=7 / 10)$ showed slight or moderate oligemia after trauma, while only three animals $(n=3 / 10)$ had a slightly hyperemic response to the mTBI. On average, the ipsilateral hemisphere showed a mild but significant CBF reduction (overall change for 15min epoch: $17.8 \% \pm 6.9 \%$ decrease $)(p=0.027)$, whereas the contralateral hemisphere showed no changes (overall change for $15-$ min epoch: $0.9 \% \pm 7.7 \%)(p=0.458)$ compared to the baseline values. The maximal reduction of CBF was observed at the peri-impact area (ipsilateral-posterior) $(24.1 \% \pm 5.1 \%$, $p<0.001$, compared to baseline).

Comparing the CBF changes between two hemispheres in time-series, the ipsilateral hemisphere had significantly lower CBF values than the contralateral hemisphere $(p=0.029)$ (Figure 3). This difference was also more pronounced at the peri-impact (ipsilateral-posterior) compared to a contralateralposterior area $(p=0.011)$. Nevertheless, the difference in CBF changes between the anterior and posterior regions of the ipsilateral hemisphere did not reach a statistical significance $(p=0.122)$ (Figure 3$)$. These results showed that after mTBI, a moderate oligemia affects the ipsilateral, but not the contralateral hemisphere, which is more marked in the areas that are directly affected by the impact.
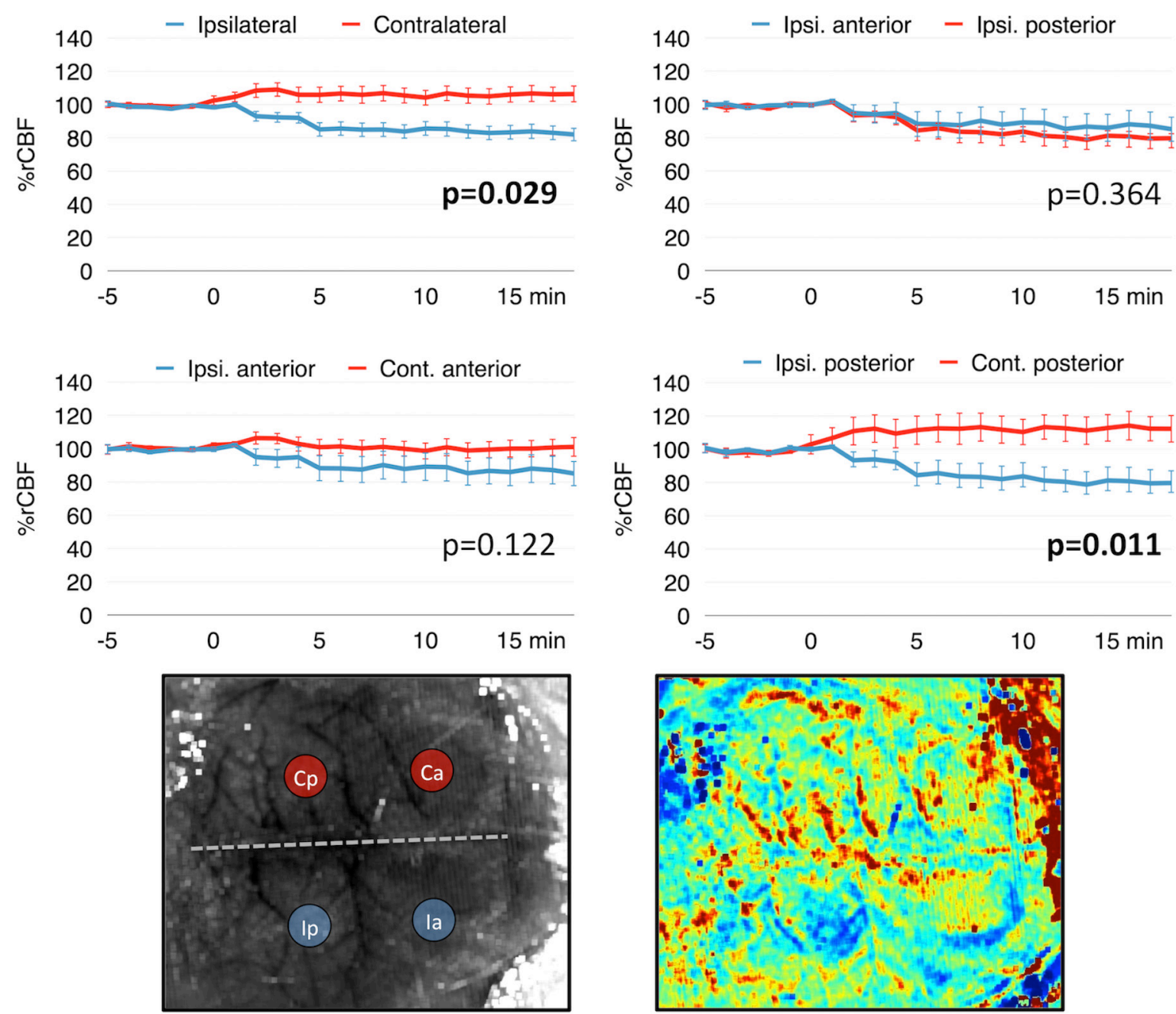

Figure 3: Post-traumatic CBF changes in the cortex. The Red and Blue dots show the regions of interest where rCBF measurements were performed. Ca: Contralateral-anterior, Cp: Contralateral-posterior, la: Ipsilateral-anterior, Ip: Ipsilateral-posterior, rCBF: regional cerebral blood flow. 


\section{Induced CSD But Not Preceding mTBI May Alter the Cortical CVR to $\mathrm{CO}_{2}$}

We studied the effects of $\mathrm{MTBI}$ and induced CSD, both separately and in sequence, on the cortical CVR during the $5 \%$ $\mathrm{CO}_{2}$ inhalation (Figure 4A). No significant difference was found in the measured CVR at the whole cerebral convexity between the sham and mTBI groups $(19.1 \% \pm 3.2 \%$ vs. $21.5 \% \pm 3.1 \%)$. The separate analyses of the two hemispheres revealed no significant difference between the ipsilateral and contralateral hemispheres within the mTBI group $(21.2 \% \pm 2.8 \%$ vs. $21.7 \%$ $\pm 3.3 \%, p=0.902)$. These results show that mTBI itself does not significantly alter the CVR (Figure 4C).

Later, we tested the effects of induced CSD following mTBI in the ipsilateral hemisphere CVR. A trend was found toward an increased overall CVR in the trauma group, particularly in the ipsilateral hemisphere, but without significant difference. However, CVR disturbance seems to have a temporal relationship with the post-CSD oligemia period. The temporal profile of the CVR showed attenuation of CVR within $30 \mathrm{~min}$ of the post-CSD period $(15.6 \% \pm 2.9 \%)$, while it gradually recovers and even overshoots the baseline CVR values toward the end of the sustained oligemia period (30-60 min) $(34.7 \% \pm$ $4.5 \%$ ) (Figure 4B). These CVR changes were similar to those seen during the CSD course in the sham animals $14.9 \%$ $\pm 1.8 \%$ in the early and $32.1 \pm 5.5 \%$ in the late post-CSD period). Therefore, we concluded that the temporal profile of the induced CSD, but not the preceding mTBI itself, has an impact on CVR.

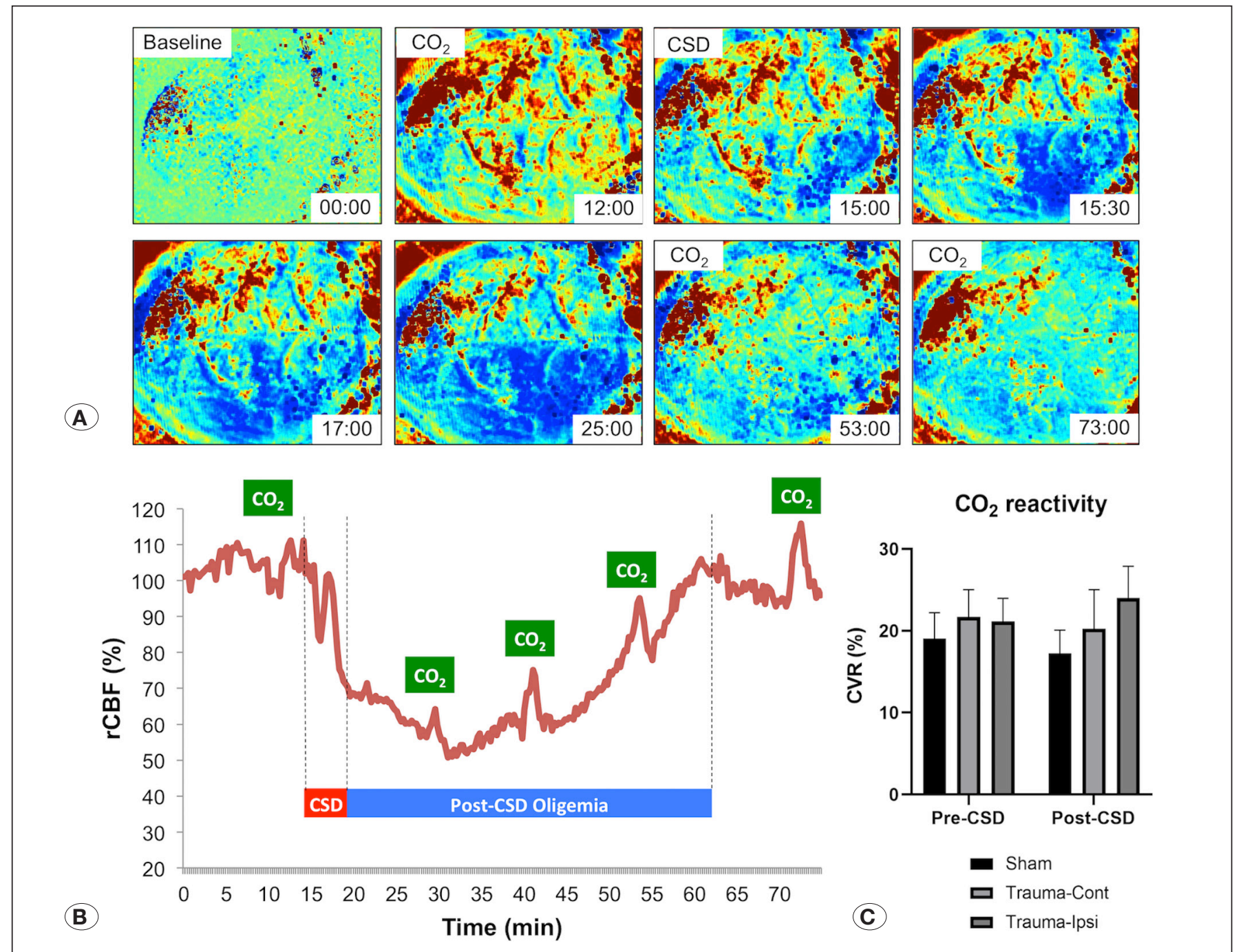

Figure 4: Cerebrovascular reactivity changes. A) Colored images show full-field cortical CBF changes at different time points (min: sec) from the onset of trauma. Note the spreading wave of oligemia from anterior to posterior after CSD induction with a pinprick. B) Graph shows CBF responses to $5 \% \mathrm{CO}_{2}$ inhalation episodes superimposed on the post-CSD oligemia period. Recovery of CVR through time is evident in this animal. C) A trend was found toward increased CVR in the ipsilateral hemisphere during the overall post-CSD period in the $\mathrm{mTBI}$ group; however, the difference was not significant. This is possibly due to the negating effect of diminished CVR in the acute phase on the increased CVR in the late phase of the post-CSD period. 


\section{Preceding mTBI Does not Alter the Cortical Hemodynamic Response to an Induced Spreading Depolarization}

We analyzed whether cortical hemodynamic responses to the CSD induction are altered after mTBI. This study used the $\mathrm{MTBI}$ paradigm that triggered CSD only in two animals $(16.7 \%)$, thus we decided to exclude them and compare hemodynamic properties of the CSDs induced by the pinprick model in the $\mathrm{mTBI}(\mathrm{n}=10$, without previous CSD) and sham groups $(n=6)$. The $\mathrm{LSCl}$ results showed that normal vascular response to CSD in mice was well-preserved after mTBI. Neither temporal profile (phase durations) nor CBF changes significantly differed between the two groups (Figure 5 and 6). These results demonstrate that $\mathrm{mTBl}$-induced moderate oligemia is insufficient to generate an inverse-hemodynamic response as in severe TBI.

\section{Uncomplicated mTBI Does not Cause Structural Damage}

Hematoxylin-eosin staining showed no structural histopathological changes. Fibrin staining was also negative for microthrombi in the mTBI group (Figure 7).

\section{DISCUSSION}

In this study, early CBF changes, CVR, and vascular responses that are associated with CSD were investigated after mild blunt head trauma in mice. Here we demonstrated that following an uncomplicated $\mathrm{mTBI}$ with no skull fracture or intracranial hemorrhage (clinically similar to a concussion) in the experimental mouse model, (i) spatiotemporal dynamics of the cortical CBF changes can be consistently examined with LSCl through the intact skull, (ii) spontaneous CSDs occur in relatively low frequency (16.7\%), (iii) a mild transient oligemia occurs in the ipsilateral, but not contralateral,

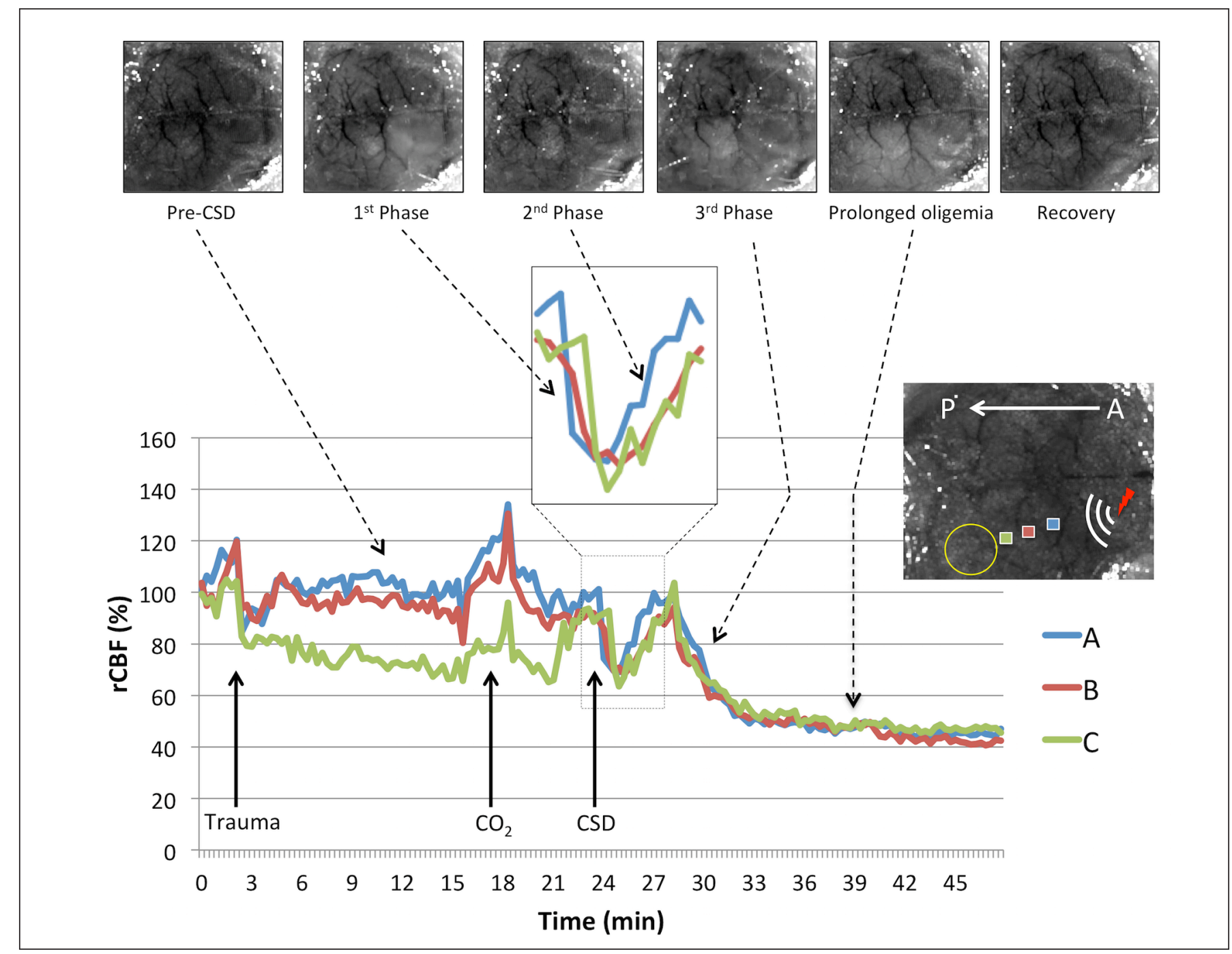

Figure 5: Temporal course of CSD. Typically, CSD is characterized by a very brief initial hyperemic phase (not shown here), followed by hypoperfusion phase (first phase), recovery/hyperemic phase (second phase), and second hypoperfusion phase (third phase), followed by a prolonged oligemia phase. Note that CSD is induced with a pinprick through a frontal burr hole, thus propagates from the anterior to the posterior direction. 

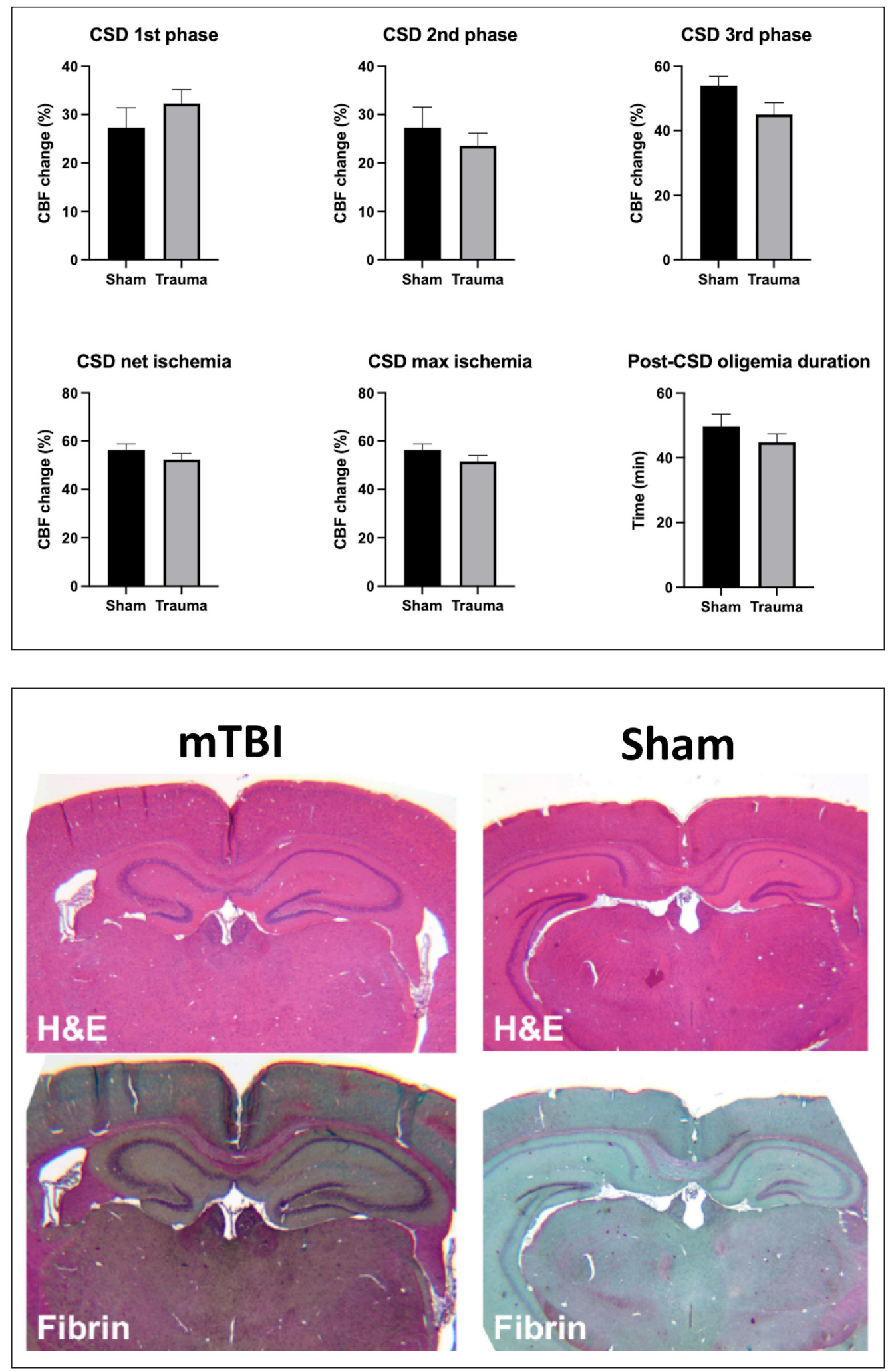

Figure 6: Cerebral blood flow changes during various phases of CSD and post-CSD oligemia duration between the trauma and sham groups. No significant differences were found between the two groups in any of the measured parameters.

Figure 7: Histopathologic examination showed no structural damage or microthrombi formation in the mTBI vs. sham group. 
hemisphere, iv) preceding minor blunt trauma alone does not impair CVR unless it causes or is complicated with a CSD, (v) CVR alterations may be temporally coupled with the recovery of post-CSD oligemia period, and vi) preceding mTBI does not alter normal vascular responses to a pinprick-induced CSD.

\section{Feasibility of LSCI to Study CBF Changes in TBI}

$\mathrm{LSCl}$ provides information on the relative blood flow velocity using the changes in the reflection patterns depending on the speed of the erythrocytes in the vessel (1). Due to the limited penetration depth, there must be no barrier between the skull and the cerebral cortex (e.g., fracture, hemorrhage) for CBF monitoring via LSCI. Therefore, we investigated the most appropriate required trauma severity to detect damage as demonstrated by LSCI by changing the height. Moderate to severe head trauma with structural damage, such as skull fracture and hemorrhages, made the use of LSCI impossible. However, we successfully managed to study CBF dynamics in animals with mTBI. This study used a trauma protocol that produced a minor blunt head trauma comparable to that of uncomplicated mTBI or concussion in humans. Hence, the described experimental protocol and imaging setup can be used to study local and global CBF dynamics in the setting of $\mathrm{mTBI}$ in mice. This study is one of the very few investigations that showed a promising $\mathrm{LSCl}$ role in mild TBI experiments. Recently, Wang et al. (43) used LSCI during craniotomy for an acute subdural hematoma in a rat model, and Lynch et al. (32) used it in a mouse model of repetitive $\mathrm{mTBI}$.

\section{Early CBF Changes After mTBI}

We investigated the CBF changes immediately after trauma. CBF decreased by up to $15 \%-20 \%$ in the ipsilateral hemisphere while no significant change was evident in the contralateral hemisphere. In the peri-impact area, the reduction was even larger (20\%-25\%). Previous studies have demonstrated that moderate ischemia is observed around the impact zone and this pericontusional ischemia may persist for weeks (8). Our findings confirm that a hypoperfusion response occurs within minutes after trauma (6,33). This hypoperfusion response probably varies depending on trauma severity, such that CBF decreases by $20 \%-25 \%$ following mild/moderate trauma while up to $50 \%-60 \%$ (ischemic levels) after severe TBI $(8,39)$. The CBF threshold for the development of infarct in trauma $(15 \mathrm{ml} / 100 \mathrm{~g} / \mathrm{min})$ is higher than that in stroke $(5-8.5$ $\mathrm{ml} / 100 \mathrm{~g} / \mathrm{min}$ ) also indicates the clinical importance of the degree of hypoperfusion in various settings. Several studies revealed that focal or global ischemia is common after TBI and cerebral hypoperfusion adversely affects the clinical outcomes (45). Vascular responses after TBI usually have three distinct phases as follows: early hypoperfusion (first 24 h), hyperperfusion (1-3 days), and late vasospasm (4-15 days) (27). In the hyperacute period, hyperperfusion may occur at first or may occur following post-traumatic ischemia. In our experiments, a slight hyperemic response was observed in a portion of animals $(n=3 / 10)$, whereas oligemic response in the majority of the cohort. The main determinant of the CBF response is probably the achieved metabolic demand with the blood flow as decreased blood flow may result in ischemia while increased blood flow may result in elevated intracranial pressure due to vasodilation (45). Late vasospasm is often associated with traumatic subarachnoid hemorrhages. Despite their similarities with aneurysmal subarachnoid hemorrhages, they have mechanistic and clinical differences. Post-traumatic radiological vasospasm occurs in $20 \%-40 \%$ of cases but is usually clinically subtle (27).

Early CBF changes after mTBI have not been extensively studied in the literature. In the clinical setting, this is challenging because the benign course of $\mathrm{mTBI}$ or concussion usually precludes any neuroimaging. Additionally, hyperacute imaging for CBF changes may be technically impossible due to the nature of the clinical condition. Imaging studies showed prolonged (from days to weeks) hypoperfusion in most patients with a concussion but an unaltered or even increased CBF response in an early post-concussion period in some patients $(10,11,28,35,44)$.

Recently, new imaging technologies studied cerebral vasculature and blood flow dynamics in vivo experiments in animals. Long et al. revealed that magnetic resonance imaging of rats undergoing open-skull mTBI model showed hyperacute hypoperfusion in the impact zone, which became hyperperfused in the impact core but remained hypoperfused around the impact core up to 2 days post-injury (31). Liu et al. studied LSCI in the controlled-cortical impact model of $\mathrm{TBI}$ and revealed a reduction in cerebral perfusion at the peri-contusional area in the acute phase of TBI; however, this reduction recovered in the chronic phase of TBI. Their trauma model produced a contusion or brain lesion, therefore did not mimic a true concussion (30). Han et al. recently revealed that $\mathrm{mTBI}$ caused acute vascular spasm, reduction of CBF, and formation of microthrombi in the acute stage, using twophoton imaging in mice (22). However, the association of their detected injury paradigm hyperacute CBF changes with a CSD is unclear, which has been possibly induced by closed head trauma itself.

\section{CVR after $\mathrm{mTBI}$}

Cerebrovascular resistance is under the significant influence of partial pressure of carbon dioxide $\left(\mathrm{PaCO}_{2}\right)$, referred to as cerebral $\mathrm{CO}_{2}$ reactivity. $\mathrm{CO}_{2}$ inhalation is frequently used in CVR evaluation as changes in $\mathrm{CO}_{2}$ lead to robust CBF alterations such that for each $1 \mathrm{~mm} \mathrm{Hg}$ increase in $\mathrm{PaCO}_{2}$ (range: $25-75 \mathrm{~mm} \mathrm{Hg}$ ), CBF increases by $2 \%-4 \% . \mathrm{CO}_{2}$ is the most potent physiologic cerebral vasodilator. Hypercapnia results in vasodilation in cerebral vessels thus increasing $\mathrm{CBF}$, while hypocapnia causes vasoconstriction and result in CBF reduction. These changes occur promptly and transiently. CBF change starts within seconds and reaches a plateau in approximately $2 \mathrm{~min}$. Generally, $\mathrm{CO}_{2}$ reactivity is better preserved compared to cerebral autoregulation (45). Vascular reactivity is impaired in the early stages in patients with severe $\mathrm{TBI}$ with a poor prognosis but is generally intact in many other patients and this response is even utilized to reduce the intracranial pressure (ICP) $(9,17,37)$. In mTBI, it is usually preserved; however, some studies indicate an altered CVR as well $(13,28)$. 


\section{CVR after CSD}

Experimental studies revealed that vascular reactivity to $\mathrm{pH}$ and $\mathrm{K}^{+}$, adenosine, and bradykinin is impaired for up to 75 min following CSD. Additionally, CSD disrupts neurovascular coupling. Further, studies with different species have also demonstrated that $\mathrm{CO}_{2}$ reactivity deteriorates for $20 \mathrm{~min}$ to $12 \mathrm{~h}$ starting approximately from $15 \mathrm{~min}$ after CSD. This deterioration is caused by nitric oxide-mediated mechanisms (2). Interestingly, in our study, vascular reactivity was partially impaired, particularly during the first 15-30 min of the postCSD ischemia period, and toward the end of this prolonged oligemia period, it recovered to and even surpassed the pre-CSD values. Further studies can better characterize the spatiotemporal dynamics of CVR changes in the relationship with the temporal course of post-CSD oligemia.

\section{CSD after TBI}

CSD or depression is a slowly propagating depolarizing wave, which causes substantial neurovascular and cellular alterations, as well as suppression of cortical electrical activity. It is implicated in a wide range of disorders from migraine to stroke, TBI to subarachnoid hemorrhages. The presence of CSD in TBI and its association with poor prognosis have been demonstrated in patients with severe TBI $(23,40)$. The incidence of CSD in patients with severe TBI is up to $50 \%-$ $60 \%$ (18). Vascular responses to CSD waves may vary depending on the ischemic conditions of the tissue. A hyperemic response is seen in healthy tissue; however, an oligemic response, called an inverse-hemodynamic response, is observed in ischemic tissue, thus further deepening the existing ischemia $(2,16)$. Additionally, frequent repetitive CSDs cause gradual brain glucose depletion and thus a massive energy imbalance (19). Prospective, multicenter, and observational studies by the COSBID group revealed that CSDs have a poor prognostic effect on 6-month mortality and morbidity outcomes, especially when accompanied by an inverse-hemodynamic response $(23,25)$. Prevention of recurrent CSDs is postulated to improve the outcomes in patients with TBI (41). Many of the currently used anesthetics reduce the incidence and amplitudes of CSDs; however, ketamine seems the most effective agent in this regard (24).

The relationship between moderate/severe or penetrating TBI and CSD is well established; however, there is not much evidence about its occurrence after a concussion or mild TBI. Recently, Bouley et al.(5) revealed that while no CSD was observed in mice that are subjected to a 25-g weight drop, $58.9 \%$ of mice subjected to a $50-\mathrm{g}$ weight drop developed CSD. Additionally, the incidence of CSDs after mTBI was linked with histological and behavioral outcomes, which may indicate their role in post-concussive symptoms. Pacheco et al. provided electrophysiological, CBF, and behavioral evidence for CSD occurrence after an mTBI in animals (36). We also noted that CSDs can be triggered even with a very mild blunt TBI protocol that is used in our experiments.

\section{CSD-Associated CBF Changes}

A few studies investigated the vascular responses to CSD in experimental TBI models. The study of Von Baumgarten et al., in the mouse TBI model created by the controlled-cortical impact method, observed spontaneous CSDs immediately after trauma and a decreased CBF in both hemispheres. However, in the following hours, CSD and accompanying vascular changes were limited to the ipsilateral hemisphere. Additionally, potassium-induced repetitive CSDs did not increase either the ICP or the contusion volume. Therefore, the authors concluded that CSD does not cause permanent brain damage as long as perfusion and oxygenation are normal (42). In our study, CSD was initially induced in only two animals due to mild trauma severity; however, vascular responses to a subsequent pinprick-induced CSD were preserved. As the effects of CSD are similar to those in normal animals and the absence of inverse-hemodynamic response indicates that serious disruptions in CBF regulation do not occur in mTBI. The absence of cell damage and microthrombus formation in the sampled brain sections also support this notion.

\section{Microcirculatory Changes in mTBI}

Some studies revealed microvascular and neuronal abnormalities at the microscopic level in the early stages of trauma. In one study, mechanical damage to cortical microvessels, blood-brain barrier disruption, and intravascular thrombocyte aggregation was observed as early as 15 min after TBI (15). Han et al. also revealed microthrombus formation in the acute period after mTBI. From this point of view, the lack of significant findings in our study may be due to the very mild trauma severity (22). More detailed histopathological, immunohistochemical, and ultrastructural studies are needed to closely examine the effects of microcirculatory deteriorations at the tissue and cell levels in the early period after TBI.

\section{Strengths and Limitations}

The strengths of our study include the ability to map (spatial) and monitor (temporal) the dynamics of cortical CBF with $\mathrm{LSCl}$ after $\mathrm{mTBI}$, revealing the necessary conditions and methodological procedures to observe CBF changes with LSCI in experimental TBI setting and imaging through the intact skull, as well as confirming mild head trauma by both gross macroscopic, histopathological characteristics, and CBF alterations. The presence of artifacts that reduce LSCI image quality, the relatively short imaging and follow-up duration, and the inability to investigate the temporal relationship of $\mathrm{CO}_{2}$ application with CSD are the predominant limitations in our study. Additionally, early and late vascular changes could be studied with various in vivo and ex vivo analyses. This experimental model and imaging setup are used to study uncomplicated $\mathrm{mTBI}$ or concussion in mice as supplemented with other available research tools. Moreover, $\mathrm{LSCl}$ is capable of detecting CSDs immediately after mTBI; therefore, it can be used to differentiate CSD-induced changes from mTBI-induced (non-CSD associated) changes in the cerebral cortex of mice.

\section{CONCLUSION}

This study revealed that in a mild TBI model in mice, a transient moderate oligemia occurred in the ipsilateral hemisphere, particularly in the peri-impact area, immediately after the 
trauma. This paradigm generates CSD in a small portion of animals, but in those without prior CSD, and MTBI itself did not alter normal vascular responses to pinprick-induced CSD. CVR to $\mathrm{CO}_{2}$ was preserved after $\mathrm{mTBI}$ in the absence of CSD. Nevertheless, mild vascular reactivity reduction was detected during the first half of the prolonged post-CSD oligemia period of the CSD; whereas, the recovery and even increased vascular reactivity was observed toward the end of the postCSD oligemia period. Further studies are needed to better characterize the mechanisms that underly these changes and investigate the earliest CBF changes related to potential structural and functional changes ensuing mTBI.

\section{ACKNOWLEDGMENTS}

Preparation for publication of this article is partly supported by Turkish Neurosurgical Society.

The authors would like to thank Dr. Damla Hanalioglu for her critical review and constructive comments.

\section{AUTHORSHIP CONTRIBUTION}

The authors confirm contribution to the paper as follows:

Study conception and design: $\mathrm{SH}, \mathrm{MM}$

Data collection: $\mathrm{SH}, \mathrm{TH}, \mathrm{II}, \mathrm{CCA}$

Analysis and interpretation of results: $\mathrm{SH}$, I

Draft manuscript preparation: $\mathrm{SH}$

Critical revision of the article: MM, II, MY

Other (study supervision, funding, materials etc.): $\mathrm{MM}, \mathrm{SH}$

All authors (SH, TH, II, CCA, MY, MM) reviewed the results and approved the final version of the manuscript.

\section{REFERENCES}

1. Ayata C, Dunn AK, Gursoy-Ozdemir Y, Huang Z, Boas DA, Moskowitz MA: Laser speckle flowmetry for the study of cerebrovascular physiology in normal and ischemic mouse cortex. J Cereb Blood Flow Metab 24:744-755, 2004

2. Ayata C, Lauritzen M: Spreading depression, spreading depolarizations, and the cerebral vasculature. Physiol Rev 95: 953-993, 2015

3. Baethmann A, Eriskat J, Stoffel M, Chapuis D, Wirth A, Plesnila N: Special aspects of severe head injury: Recent developments. Curr Opin Anaesthesiol 11:193-200, 1998

4. Barkhoudarian G, Hovda DA, Giza CC: The molecular pathophysiology of concussive brain injury. Clin Sports Med 30: 33-48, vii-iii, 2011

5. Bouley J, Chung DY, Ayata C, Brown RH, Henninger $\mathrm{N}$ : Cortical spreading depression denotes concussion injury. J Neurotrauma 36:1008-1017, 2019

6. Bouma GJ, Muizelaar JP, Choi SC, Newlon PG, Young HF: Cerebral circulation and metabolism after severe traumatic brain injury: The elusive role of ischemia. J Neurosurg 75: 685693, 1991
7. Bramlett HM, Dietrich WD: Long-term consequences of traumatic brain injury: Current status of potential mechanisms of injury and neurological outcomes. J Neurotrauma 32:18341848,2015

8. Bullock R, Sakas D, Patterson J, Wyper D, Hadley D, Maxwell W, Teasdale GM: Early post-traumatic cerebral blood flow mapping: Correlation with structural damage after focal injury. Acta Neurochir Suppl (Wien) 55:14-17, 1992

9. Carmona Suazo JA, Maas Al, van den Brink WA, van Santbrink $\mathrm{H}$, Steyerberg EW, Avezaat CJ: CO2 reactivity and brain oxygen pressure monitoring in severe head injury. Crit Care Med 28:3268-3274, 2000

10. Churchill NW, Hutchison MG, Graham SJ, Schweizer TA: Symptom correlates of cerebral blood flow following acute concussion. Neuroimage Clin 16:234-239, 2017

11. Churchill NW, Hutchison MG, Richards D, Leung G, Graham SJ, Schweizer TA: The first week after concussion: Blood flow, brain function and white matter microstructure. Neuroimage Clin 14:480-489, 2017

12. Coles JP, Fryer TD, Smielewski P, Chatfield DA, Steiner LA, Johnston AJ, Downey SPMJ, Williams GB, Aigbirhio F, Hutchinson PJ, Rice K, Carpenter TA, Clark JC, Pickard JD, Menon DK: Incidence and mechanisms of cerebral ischemia in early clinical head injury. J Cereb Blood Flow Metab 24: 202-211, 2004

13. da Costa L, van Niftrik CB, Crane D, Fierstra J, Bethune A: Temporal profile of cerebrovascular reactivity impairment, gray matter volumes, and persistent symptoms after mild traumatic head injury. Front Neurol 7:70, 2016

14. Dewan MC, Rattani A, Gupta S, Baticulon RE, Hung YC, Punchak M, Agrawal A, Adeleye AO, Shrime MG, Rubiano AM, Rosenfeld JV, Park KB: Estimating the global incidence of traumatic brain injury. J Neurosurg, 2018 (Online ahead of print)

15. Dietrich WD, Alonso O, Halley M: Early microvascular and neuronal consequences of traumatic brain injury: A light and electron microscopic study in rats. J Neurotrauma 11:289301, 1994

16. Dreier JP: The role of spreading depression, spreading depolarization and spreading ischemia in neurological disease. Nat Med 17:439-447, 2011

17. Enevoldsen EM, Jensen FT: Autoregulation and $\mathrm{CO} 2$ responses of cerebral blood flow in patients with acute severe head injury. J Neurosurg 48:689-703, 1978

18. Fabricius M, Fuhr S, Bhatia R, Boutelle M, Hashemi P, Strong AJ, Lauritzen M: Cortical spreading depression and periinfarct depolarization in acutely injured human cerebral cortex. Brain 129:778-790, 2006

19. Feuerstein D, Manning A, Hashemi P, Bhatia R, Fabricius M, Tolias C, Pahl C, Ervine M, Strong AJ, Boutelle MG: Dynamic metabolic response to multiple spreading depolarizations in patients with acute brain injury: An online microdialysis study. J Cereb Blood Flow Metab 30:1343-1355, 2010

20. Forouzan A, Barzegari H, Hosseini O, Delirrooyfard A: The diagnostic competence of glial fibrillary acidic protein in mild traumatic brain injury and its prognostic value in patient recovery. Turk Neurosurg 31:355-360, 2021 
21. Ghajar J: Traumatic brain injury. Lancet 356:923-929, 2000

22. Han X, Chai Z, Ping X, Song LJ, Ma C, Ruan Y, Jin X: In vivo two-photon imaging reveals acute cerebral vascular spasm and microthrombosis after mild traumatic brain injury in mice. Front Neurosci 14:210, 2020

23. Hartings JA, Bullock MR, Okonkwo DO, Murray LS, Murray GD, Fabricius M, Maas Al, Woitzik J, Sakowitz O, Mathern $B$, Roozenbeek B, Lingsma H, Dreier JP, Puccio AM, Shutter LA, Pahl C, Strong AJ, Co-Operative Study on Brain Injury Depolarisations: Spreading depolarisations and outcome after traumatic brain injury: A prospective observational study. Lancet Neurol 10:1058-1064, 2011

24. Hertle DN, Dreier JP, Woitzik J, Hartings JA, Bullock R, Okonkwo DO, Shutter LA, Vidgeon S, Strong AJ, Kowoll C, Dohmen C, Diedler J, Veltkamp R, Bruckner T, Unterberg AW, Sakowitz OW, Cooperative Study of Brain Injury Depolarizations (COSBID): Effect of analgesics and sedatives on the occurrence of spreading depolarizations accompanying acute brain injury. Brain 135:2390-2398, 2012

25. Hinzman JM, Andaluz N, Shutter LA, Okonkwo DO, Pahl C, Strong AJ, Dreier JP, Hartings JA: Inverse neurovascular coupling to cortical spreading depolarizations in severe brain trauma. Brain 137:2960-2972, 2014

26. Inoue Y, Shiozaki T, Tasaki O, Hayakata T, Ikegawa H, Yoshiya $\mathrm{K}$, Fujinaka T, Tanaka H, Shimazu T, Sugimoto H: Changes in cerebral blood flow from the acute to the chronic phase of severe head injury. J Neurotrauma 22:1411-1418, 2005

27. Kramer DR, Winer JL, Pease BAM, Amar AP, Mack WJ: Cerebral vasospasm in traumatic brain injury. Neurol Res Int 2013:415813, 2013

28. Len TK, Neary JP: Cerebrovascular pathophysiology following mild traumatic brain injury. Clin Physiol Funct Imaging 31:8593, 2011

29. Li W, Watts L, Long J, Zhou W, Shen Q, Jiang Z, Li Y, Duong TQ: Spatiotemporal changes in blood-brain barrier permeability, cerebral blood flow, T2 and diffusion following mild traumatic brain injury. Brain Res 1646:53-61, 2016

30. Liu H, He J, Zhang Z, Liu L, Huo G, Sun X, Cheng C: Evolution of cerebral perfusion in the peri-contusional cortex in mice revealed by in vivo laser speckle imaging after traumatic brain injury. Brain Res 1700:118-125, 2018

31. Long JA, Watts LT, Li W, Shen Q, Muir ER, Huang S, Boggs RC, Suri A, Duong TQ: The effects of perturbed cerebral blood flow and cerebrovascular reactivity on structural MRI and behavioral readouts in mild traumatic brain injury. J Cereb Blood Flow Metab 35:1852-1861, 2015

32. Lynch CE, Eisenbaum M, Algamal M, Balbi M, Ferguson S, Mouzon B, Saltiel N, Ojo J, Diaz-Arrastia R, Mullan M, Crawford F, Bachmeier C: Impairment of cerebrovascular reactivity in response to hypercapnic challenge in a mouse model of repetitive mild traumatic brain injury. J Cereb Blood Flow Metab 41:1362-1378, 2021
33. Marion DW, Darby J, Yonas H: Acute regional cerebral blood flow changes caused by severe head injuries. J Neurosurg 74: 407-414, 1991

34. Martin NA, Patwardhan RV, Alexander MJ, Africk CZ, Lee $\mathrm{JH}$, Shalmon E, Hovda DA, Becker DP: Characterization of cerebral hemodynamic phases following severe head trauma: Hypoperfusion, hyperemia, and vasospasm. J Neurosurg 87: 9-19, 1997

35. Meier TB, Bellgowan PSF, Singh R, Kuplicki R, Polanski DW, Mayer AR: Recovery of cerebral blood flow following sportsrelated concussion. JAMA Neurol 72:530-538, 2015

36. Pacheco JM, Hines-Lanham A, Stratton C, Mehos CJ, McCurdy KE, Pinkowski NJ, Zhang $\mathrm{H}$, Shuttleworth CW, Morton RA: Spreading depolarizations occur in mild traumatic brain injuries and are associated with postinjury behavior. eNeuro 6(6):ENEURO.0070-19.2019, 2019

37. Sahuquillo J, Munar F, Baguena M, Poca MA, Pedraza S, Rodríguez-Baeza A: Evaluation of cerebrovascular CO2reactivity and autoregulation in patients with post-traumatic diffuse brain swelling (diffuse injury III). Acta Neurochir Suppl 71:233-236, 1998

38. Seifert J: Incidence and economic burden of injuries in the United States. J Epidemiol Community Health 61:926, 2007

39. Shen Y, Kou Z, Kreipke CW, Petrov T, Hu J, Haacke EM: In vivo measurement of tissue damage, oxygen saturation changes and blood flow changes after experimental traumatic brain injury in rats using susceptibility weighted imaging. Magn Reson Imaging 25:219-227, 2007

40. Strong AJ, Fabricius M, Boutelle MG, Hibbins SJ, Hopwood SE, Jones R, Parkin MC, Lauritzen M: Spreading and synchronous depressions of cortical activity in acutely injured human brain. Stroke 33:2738-2743, 2002

41. Strong AJ, Hartings JA, Dreier JP: Cortical spreading depression: An adverse but treatable factor in intensive care? Curr Opin Crit Care 13:126-133, 2007

42. von Baumgarten L, Trabold R, Thal S, Back T, Plesnila N: Role of cortical spreading depressions for secondary brain damage after traumatic brain injury in mice. J Cereb Blood Flow Metab 28:1353-1360, 2008

43. Wang C, Xian L, Chen X, Li Z, Fang Y, Xu W, Wei L, Chen W, Wang S: Visualization of cortical cerebral blood flow dynamics during craniotomy in acute subdural hematoma using laser speckle imaging in a rat model. Brain Res 1742:146901, 2020

44. Wang Y, Nelson LD, LaRoche AA, Pfaller AY, Nencka AS, Koch KM, McCrea MA: Cerebral blood flow alterations in acute sport-related concussion. J Neurotrauma 33:1227-1236, 2016

45. Werner C, Engelhard K: Pathophysiology of traumatic brain injury. Br J Anaesth 99:4-9, 2007 